Annales Academiæ Scientiarum Fennicæ

Series A. I. Mathematica

Volumen 15, 1990, 283-291

\title{
THE ESSENTIAL STATE DIAGRAM OF A LINEAR OPERATOR
}

\author{
R.W. Cross
}

Given an operator $T: D(T) \subset X \rightarrow Y$ where $X$ and $Y$ are normed spaces, we call $T$ on $F_{+}$-operator [C2] if there exists a subspace $E$ of finite codimension in $X$ for which $(T / E)^{-1}$ exists and is continuous. We investigate properties of $T$ related to the quantities $\alpha(T), \beta(T), \bar{\beta}(T)$ and the property $T \in F_{+}$and construct a state diagram, called the essential state diagram, analogous to the Taylor-Halbert model [TH] (see also [G1], [G2] and [G3]); the latter will be referred to as the THG state diagram. A consequence is the following observation: $T$ is an $F_{+}$operator if and only if its adjoint $T^{\prime}$ is a $\varphi_{-}$-operator (Corollary 1.17). Various other state diagrams have appeared in the literature. The reader may consult the survey monograph of V.M. Onieva [O] for further references.

Let $X, Y, Z, \ldots$ denote normed linear spaces. The completion of $X$ will be denoted by $\tilde{X}$. The class of linear transformations (henceforth called "operators") $T$ defined on a linear subspace $D(T)$ of $X$ with range contained in $Y$ is denoted by $L(X, Y)$. The range and null space of $T$ are denoted by $R(T)$ and $N(T)$ respectively. The restriction of $T$ to a linear subspace $M$ of $X$ is denoted by $T / M$; note that $T / M=T / M \cap D(T)$. The operator $T$ is called bounded if $T$ is continuous and $D(T)=X . T$ is called closed if its graph $\{(x, T x): x \in D(T)\}$ is a closed subset of $X \times Y$. Let $X_{T}$ be the space $D(T)$ normed by $\|x\|_{T}=\|x\|+\|T x\|$. The graph operator $G_{T}$ of $T$ is the operator in $L\left(X_{T}, X\right)$ defined by $G_{T} x=x$ $\left(x \in X_{T}\right)$. We write $G=G_{T}$. Clearly $T G$ is a bounded operator in $L\left(X_{T}, Y\right)$. Let $E$ be a linear subspace of $X$. Following Pietsch $[\mathrm{P}]$ we denote by $J_{E}^{X}$ and $Q_{E}^{X}$ respectively the natural injection of $E$ into $X$ and the natural quotient map of $X$ onto $X / E$. The adjoint $T^{\prime}$ of $T$ is defined by $T^{\prime}=\left(T J_{D(T)}^{X}\right)^{\prime}$ where the righthand side is the conjugate defined in the usual sense [G3; 50]. Note that $T^{\prime} \in L\left(Y^{\prime}, D(T)^{\prime}\right)$. We clearly have $\left(J_{E}^{X}\right)^{\prime}=Q_{E^{\perp}}^{X^{\prime}}$, and if $E$ is closed then $\left(Q_{E}^{X}\right)^{\prime}=J_{E^{\perp}}^{X^{\prime}}$. It is evident that the state diagrams II.3.14 and II.4.11 of [G3] are valid in the general case. Given two linear subspaces $M, N$ of $X$ auch that $M \cap N=0$ we write $M \oplus N$ for $M+N$. Let $\alpha(T)=\operatorname{dim} N(T), \beta(T)=$ codim $R(T)$ and $\bar{\beta}(T)=\operatorname{codim} \overline{R(T)} . T$ is called a $\varphi_{+}$-operator if $\alpha(T)<\infty$ and $R(T)$ is closed, and a $\varphi_{-}$-operator if $R(T)$ is closed and $\beta(T)<\infty$. If $T$ is closed and $X$ and $Y$ are Banach spaces then $T \in F_{+}$if and only if $T \in \varphi_{+}$ (Proposition 1.5). The $F_{+}$-operators retain the usual properties associated with 
the $\varphi_{+}$-operators of Gohberg-Krein [GohK] (see [C3]). Other properties of $F_{+}$operators relate to stability of complemented ranges and generalised inverses [C4], and to the Tauberian property [C5].

We classify the operators $T$ in $L(X, Y)$ as follows:

Ie: $R(T)$ is closed and $\beta(T)<\infty$;

IIe: $R(T)$ is not closed but $\bar{\beta}(T)<\infty$;

IIIe: $\bar{\beta}(T)=\infty$;

$1 e: T$ is an $F_{+}$-operator;

$2 e: T \notin F_{+}$but $\alpha(T)<\infty$;

$3 e: \alpha(T)=\infty$.

If $\beta(T)<\infty$ and $R(T)$ is closed we say that $T$ is in the state $I e$ and write $T \in I e$. If $T$ is both in state IIIe and state $2 e$ then we write $T \in I I I_{2} e$. The other cases are treated similarly. It is clear that each of the sets $\{I e, I I e, I I I e\}$ and $\{1 e, 2 e, 3 e\}$ are partitions of $L(X, Y)$. There are thus nine disjoint classes $I_{1}, \ldots, I I I_{3} e$ whose union is $L(X, Y)$. The classes should be compared to the classes $I, I I, I I I, 1,2,3$ in Goldberg [G3]. It is obvious that $I \subset I e, I I \subset I I e$, $I I I \supset I I I e$ and $1 \subset 1 e, 2 \subset 1 e \cup 2 e$ and $3 \supset 3 e$.

A subspace $M$ is called a principal subspace if $M$ is closed and has finite codimension. In such a case $T / M$ is called a principal restriction of $T$.

1.1. Lemma. If $T$ has no principal restriction having a continuous inverse then there exists an infinite dimensional subspace $M$ of $D(T)$ for which $T / M$ is precompact ([Ka], [G3]; see [G3; 80]).

1.2. Lemma. If $X=M \oplus N$ where $M$ is a principal subspace then the projection of $X$ onto $M$ with null space $N$ is bounded.

1.3. Corollary. If $M$ is a principal subspace of $X$ and if $T / M$ is continuous, then $T$ is continuous.

1.4. Lemma. Let $T \in F_{+}$. Then there exists a principal subspace $M$ of $X$ for which $T / M$ has a continuous inverse.

Proof. There exists a finite codimensional subspace $E$ of $X$ such that $T / E$ has a continuous inverse. Suppose $T$ has no principal restriction having a continuous inverse. Then by Lemma 1.1 there exists an infinite dimensional subspace $N$ of $D(T)$ such that $T / N$ is precompact. But then $E \cap N$ is infinite dimensional and $T / E \cap N$ is precompact with a continuous inverse, contradicting the non-precompactness of the unit ball in an infinite dimensional normed space.

1.5. Proposition. Let $T$ be closed. Then

(a) if $X$ is complete, we have $T \in F_{+} \Longrightarrow T \in \varphi_{+}$,

(b) if $X$ and $Y$ are complete, we have $T \in \varphi_{+} \Longrightarrow T \in F_{+}$.

Proof. (a) Let $T \in F_{+}$. Then by Lemma 1.4 there exists a principal subspace $M$ and a finite dimensional subspace $F$ of $D(T)$ such that $D(T)=M \oplus F \oplus N(T)$, 
and such that $(T / M)^{-1}$ is continuous. Let $T m_{n} \rightarrow y\left(M_{n} \in M\right)$. Then $\left(m_{n}\right)$ is Cauchy, and so $m_{n} \rightarrow x$ (for an $x \in X$ ) since $X$ is complete. Thus $\left(m_{n}, T m_{n}\right) \rightarrow$ $(x, y)$ in $X \times Y$. But $T$ is closed; hence $x \in D(T)$ and $y=T x$. Since $M$ is closed in $D(T)$, we have $x \in M$. Therefore $T M$ is closed and so $R(T)=T F+T M$ is closed (see e.g. [G3; 16]). Hence $T \in \varphi_{+}$.

(b) Let $T \in \varphi_{+}$. Then $D(T)=M \oplus N(T)$ where $M$ is principal in $D(T)$ and $T M$ is closed. By the closed graph theorem $(T / M)^{-1}$ is continuous. Therefore $T \in F_{+}$.

1.6. Corollary. We have $T^{\prime} \in F_{+}$if and only if $T^{\prime} \in \varphi_{+}$.

1.7. Lemma. Let $M$ be a principal subspace of $D(T)$. Then $\left(J_{M}^{X}\right)^{\prime} T^{\prime}=$ $\left(T J_{M}^{X}\right)^{\prime}$.

Proof. Write $J=J_{M}^{X}$. We clearly have $D\left(T^{\prime}\right) \subset D\left((T J)^{\prime}\right)$ and $D\left(J^{\prime} T^{\prime}\right)=$ $D\left(T^{\prime}\right)$ since $J^{\prime}$ is bounded. Let $y^{\prime} \in D\left((T J)^{\prime}\right)$. Then $y^{\prime} T J$ is continuous on $M$, and hence on $D(T)$ since $M$ is a principal subspace of $D(T)$ by Corollary 1.3. Hence $y^{\prime} \in D\left(T^{\prime}\right)$ and the equality follows. $\square$

1.8. Lemma. Let $S \in L(X, Z)$ be a restriction of $T$ where $R(T) \subset Z \subset Y$. Then $R\left(S^{\prime}\right)=R\left(T^{\prime}\right)$.

Proof. We have $T=J_{Z}^{Y} S$, whence $T^{\prime}=S^{\prime} Q_{Z^{\perp}}^{Y^{\prime}}$ (since $J_{Z}^{Y}$ is bounded). Since $D\left(S^{\prime}\right) \subset R\left(Q_{Z^{\perp}}^{Y^{\prime}}\right)=Y^{\prime} / Z^{\perp}$ it follows immediately that $R\left(T^{\prime}\right)=R\left(S^{\prime}\right)$.

The normed space $X$ is called an operator range [C1] if it is the range of a bounded operator defined on a Banach space.

1.9. Lemma [1]. If $R_{1}$ and $R_{2}$ are disjoint complementary operator ranges in a Banach space $X$ then $R_{1}$ and $R_{2}$ are closed.

1.10. Lemma. If $T \in F_{+}$then $T^{\prime} \in \varphi_{-}$.

Proof. Let $T \in F_{+}$. By Lemma 1.4 there exists a principal subspace $E$ of $D(T)$ such that $T J_{E}^{X} \in 1$. Write $J=J_{E}^{X}$. Then $(T J)^{\prime} \in I$ by the $T H G$ state diagram [G3; 61]. Hence $J^{\prime} T^{\prime} \in I$ by Lemma 1.7. Now let $x^{\prime} \in X^{\prime}$ and choose $y^{\prime} \in Y^{\prime}$ such that $x^{\prime}+E^{\perp}=T^{\prime} y^{\prime}+E^{\perp}$. Select $w \in E^{\perp}$ so that $x^{\prime}=$ $T^{\prime} y^{\prime}+w$. This shows that $X^{\prime}=R\left(T^{\prime}\right)+E^{\perp}$. But $R\left(T^{\prime}\right)$ is an operator range (see e.g. [C1; 228]). Hence by Lemma $1.9, R\left(T^{\prime}\right)$ is closed (alternatively, apply [G3; IV.1.12]). Therefore $T^{\prime} \in \varphi_{-}$.

\subsection{Proposition.}

(i) If $T \in I e$ we have $T^{\prime} \in 1 e \cup 2 e$,

(ii) If $T \in I I e$ we have $T^{\prime} \in 1 e \cup 2 e$,

(iii) If $T \in I I I e$ we have $T^{\prime} \in 3 e$,

(iv) If $T \in 1 e$ we have $T^{\prime} \in I e$,

(v) If $T \in 2 e$ we have $T^{\prime} \in I I e \cup$ IIIe,

(vi) If $T \in 3 e$ we have $T^{\prime} \in$ IIIe. 
Proof. If $\operatorname{dim} D(T)<\infty$ all the statements (i)-(vi) are elementary or trivial. We shall therefore suppose that $\operatorname{dim} D(T)=\infty$. The statements (i), (ii) and (iii) follow immediately from the equality $N\left(T^{\prime}\right)=R(T)^{\perp}$ [G3; II.3.7], while (iv) is a restatement of Lemma 1.10. To prove (v), assume $T \in 2 e$. Then there exists an infinite dimensional subspace $E$ of $\mathrm{D}(\mathrm{T})$ for which $T J_{E}^{X}$ is precompact by Lemma 1.1. Write $J=J_{E}^{X}$. Then $(T J)^{\prime}$ is compact [G3; III.1.11]. Since $(T J)^{\prime}$ is an extension of $J^{\prime} T^{\prime}$, it follows that $J^{\prime} T^{\prime}$ is compact. Suppose (if possible) that $T^{\prime} \in I e$. Let $X^{\prime}=R\left(T^{\prime}\right) \oplus W$ where $\operatorname{dim} W<\infty$. Then

$$
J^{\prime}\left(X^{\prime}\right)=J^{\prime}\left(R\left(T^{\prime}\right) \oplus W\right) \subset R\left(J^{\prime} T^{\prime}\right)+J^{\prime} W=J^{\prime}\left(X^{\prime}\right)
$$

since $J^{\prime} \in I$. Thus $R\left(J^{\prime} T^{\prime}\right)+J^{\prime} W=J^{\prime}\left(X^{\prime}\right)=X^{\prime} / E^{\perp}$. But $R\left(T^{\prime}\right)$ is an operator range and so therefore is its continuous linear image $R\left(J^{\prime} T^{\prime}\right)$. Consequently $R\left(J^{\prime} T^{\prime}\right)$ is closed by Lemma 1.9. Since $J^{\prime} T^{\prime}$ is compact it follows that $R\left(J^{\prime} T^{\prime}\right)$ is finite dimensional. Therefore $\operatorname{dim} X^{\prime} / E^{\perp}<\infty$. Hence $\operatorname{dim} E=$ $\operatorname{dim} E^{\prime}=\operatorname{dim} X^{\prime} / E^{\perp}<\infty$, a contradiction. Therefore $T^{\prime} \notin I e$ and (v) follows. Finally to prove (vi) we have $N(T) \subset{ }^{\perp} R\left(T^{\prime}\right)$ whence $N(T)^{\perp} \supset \overline{R\left(T^{\prime}\right)}$. Hence if $T^{\prime} \in I e \cup I I e$ then $\operatorname{codim} N(T)^{\perp}<\infty$, which implies $\operatorname{dim} N(T)<\infty$ and (vi) follows. $\square$

1.12. Proposition. If $T \in I_{1} e \cup I I_{1} e$ we have $T^{\prime} \in I_{1} e$.

Proof. Let $T \in I_{1} e \cup I I_{1} e$. Then from Proposition 1.11, $T^{\prime} \in I e \cap(1 e \cup 2 e)$. Thus $\alpha\left(T^{\prime}\right)<\infty$ and $R\left(T^{\prime}\right)$ is closed, i.e., $T^{\prime}$ is a closed $\varphi_{+}$-operator. Therefore $T^{\prime} \in F_{+}$by Corollary 1.6 and the result follows. $\square$ $R\left(T^{\prime}\right)$.

1.13. Lemma. Let $\overline{R(T)} \oplus M=Y$ where $\operatorname{dim} M<\infty$. Then $R\left(\left(Q_{M}^{Y} T\right)^{\prime}\right)=$

Proof. Write $Q=Q_{M}^{Y}$. Since $\operatorname{dim} M<\infty$ there exists a bounded projection $P$ of $Y$ onto $\overline{R(T)}$ with null space $M$ by Lemma 1.2. Now let $y^{\prime} \in D\left(T^{\prime}\right)$. Then

$$
T^{\prime} y^{\prime}=\overline{y^{\prime} T}=\overline{y^{\prime} P T}=\overline{y^{\prime} P Q T}=(Q T)^{\prime}\left(y^{\prime} P\right) \in R\left((Q T)^{\prime}\right) .
$$

Thus $R\left(T^{\prime}\right) \subset R\left((Q T)^{\prime}\right)$. On the other hand since $Q$ is bounded, $(Q T)^{\prime}=T^{\prime} Q^{\prime}$. Hence $R\left((Q T)^{\prime}\right) \subset R\left(T^{\prime}\right)$. 口

1.14. Lemma. Let $S \in L(Y, Z)$ be a bounded operator. Then $S T \in F_{+}$ implies $T \in F_{+}$.

Proof. Let $S T \in F_{+}$and let $M$ be a finite dimensional subspace of $D(T)=$ $D(S T)$ for which $S T / M$ has a continuous inverse. Then $\|S\|\|T m\| \geq\|S T m\| \geq$ $c\|m\|$ for some $c>0$ and all $m \in M$. Hence $T \in F_{+} \cdot$.

\subsection{Proposition.}

(a) If $T \in I_{2} e \cup I I_{2} e$ we have $T^{\prime} \notin I I_{1} e$.

(b) If $Y$ is complete then $T \in I_{2} e$ implies $T^{\prime} \in I I I_{1} e$. 
Proof. (a) Let $T \in I_{2} e \cup I I_{2} e$ and suppose $T^{\prime} \in I I_{1} e$. Since $T^{\prime} \in 1 e, T^{\prime}$ is a $\varphi_{+}$-operator by Corollary 1.6, and hence $R\left(T^{\prime}\right)$ is closed contradicting $T^{\prime} \in I I e$. Therefore $T^{\prime} \notin I I_{1} e$.

(b) Let $Y$ be complete and let $T \in I_{2} e$. Then there exists a finite dimensional subspace $N$ of $Y$ and a closed finite codimensional subspace $E$ of $D(T)$ such that $R(T) \oplus M=Y$ and $E \oplus N(T)=D(T)$. Write $Q=Q_{M}^{Y}$ and $J=J_{E}^{X}$. It is clear that $\alpha(T J)=0$ and since $J$ is an isomorphism it follows that $T J \in 2$. Moreover $R(T J)=R(T)$. Hence $Q T J \in I_{2}$. The state diagram [G3; 61] now gives $(Q T J)^{\prime} \in I I I_{1}$ where by Lemma $1.7,(Q T J)^{\prime}=(T J)^{\prime} Q^{\prime}=J^{\prime} T^{\prime} Q^{\prime}$. Since $Q^{\prime}=J_{M^{\perp}}^{Y^{\prime}}$ we clearly have $R\left(J^{\prime} T^{\prime} Q^{\prime}\right)=R\left(J^{\prime} T^{\prime}\right)$ by Lemma 1.13 and in particular $R\left(J^{\prime} T^{\prime}\right)$ is closed. But since $T J \in I_{2} e$, Proposition 1.11 gives

$$
J^{\prime} T^{\prime} \in I I_{1} e \cup I I I_{1} e \cup I I_{2} e \cup I I I_{2} e .
$$

It follows that $J^{\prime} T^{\prime} \in I I I_{1} e$. But $J^{\prime} T^{\prime} \in 1 e$ implying that $T^{\prime} \in 1 e$ by Lemma 1.14, and the finite dimensionality of $E^{\perp}$ implies immediately that $T^{\prime} \in I I I e$. Therefore $T^{\prime} \in I I I_{1} e$. 口

1.16. Proposition. Let $Y$ be complete. Then $T \in I_{3} e$ implies $T^{\prime} \in I I I_{1} e$.

Proof. Let $T \in I_{3} e$. Then $Y=R(T) \oplus M$ where $\operatorname{dim} M<\infty$. Write $Q=Q_{M}^{Y}$. Then $Q T \in I \cap 3 e \subset I_{3}$. Since $Y$ is complete the $T H G$ state diagram [G3; 61] gives $T^{\prime} Q^{\prime}=(Q T)^{\prime} \in I I I_{1}$. But $Q^{\prime}=J_{M^{\perp}}^{Y^{\prime}}$ where $\operatorname{codim} M^{\perp}<\infty$. Therefore $T^{\prime} \in 1 e$. Hence by Lemma 1.11, $T^{\prime} \in I I I_{1} e$. व

The results of this section are summarized in the state diagram 2.7 of the next section. Note that the resulting configuration is the same as II.3.14 of [G3].

1.17. Corollary. We have $T \in F_{+}$if and only if $T^{\prime} \in \varphi_{-}$.

\section{The essential state diagram for a closed operator}

2.1. Lemma. Let $T \in L(X, Y)$ be closed and let $Q \in L(X, Y)$ be a quotient map with finite dimensional null space. Then $Q T$ is closed.

Proof. Let $\left(x_{n}, Q T x_{n}\right) \rightarrow(x, y+N)$ where $N=N(Q)$. Then there exists a sequence $w_{n} \in N$ such that $T x_{n}-y+w_{n} \rightarrow 0$. The finite dimensionality of $N$ now implies that $\left(w_{n}\right)$ is bounded: indeed if $\left(w_{n}\right)$ is unbounded then for some subsequence $w_{n}$, we have $\left\|w_{n^{\prime}}\right\| \rightarrow \infty$ and $\left(T x_{n^{\prime}}+w_{n^{\prime}}\right) /\left\|w_{n^{\prime}}\right\| \rightarrow 0$. Since $\left(w_{n^{\prime}} /\left\|w_{n^{\prime}}\right\|\right)$ is bounded in the finite dimensional space $N$ there exists a subsequence $\left(w_{n^{\prime \prime}}\right)$ of $\left(w_{n^{\prime}}\right)$ and $k \in N$ such that $w_{n^{\prime \prime}} /\left\|w_{n^{\prime \prime}}\right\| \rightarrow k$. But then $T x_{n^{\prime \prime}} /\left\|w_{n^{\prime \prime}}\right\| \rightarrow-k$ and $x_{n^{\prime \prime}} /\left\|w_{n^{\prime \prime}}\right\| \rightarrow 0$. Since $T$ is closed, this implies that $k=0$, contradicting $\|k\|=1$. Therefore $\left(w_{n}\right)$ is bounded. Passing to a subsequence if necessary we may suppose that $w_{n}$ is convergent. Let $w=\lim w_{n}$. Then $T x_{n} \rightarrow y-w$. Since $T$ is closed we now have $x \in D(T)$ and $T x=y-w$. Therefore $Q T x=y$, showing that $Q T$ is closed. $\square$ 
2.2. Theorem [2]. Let $T \in F_{+}(X, Y)$ and $S \in F_{+}(Y, Z)$. Then $S T \in F_{+}$ whenever $S T \neq 0$.

Proof. See [C2; 2.12 and 2.13]. ㅁ

2.3. Lemma. Let $T$ be closed and $X$ complete and let $R\left(T^{\prime}\right)$ be closed. Then $R(T)$ is closed.

Proof. Let $X$ be complete and let $T_{0} \in L(X, \overline{R(T)})$ be an astriction of $T$. Then $T_{0}$ is closed, and $R\left(T_{0}^{\prime}\right)=R\left(T^{\prime}\right)$ by Lemma 1.8. Thus $T_{0}^{\prime}$ has a continuous inverse by the closed graph theorem. From the $T H G$ state diagram for closed operators [G3;66] we see that $T_{0} \in I_{1} \cup I_{3}$. Thus $T_{0}$ is surjective, i.e. $R(T)=R\left(T_{0}\right)=\overline{R(T)}$

2.4. Corollary. Let $T$ be closed and let $X$ be complete. Then $T \in I I e$ implies $T^{\prime} \notin 1 e$.

Proof. Let $T \in I I e$. Then $R(T)$ is not closed and hence $R\left(T^{\prime}\right)$ is not closed by Lemma 2.3. Consequently $T^{\prime} \notin 1 e$. $\square$

2.5. Proposition. Let $T$ be closed and let $X$ be complete. Then $T \in I_{2} e$ implies $T^{\prime} \notin 1 e$.

Proof. Let $T \in I_{2} e$ and let the operators $Q$ and $J$ be as in the proof of Proposition 1.15. Then $Q T J \in I_{2}$ and $Q T J \in L(E, Y / M)$ where $E$ is complete. Moreover $Q T J$ is closed by Lemma 2.1. By the $T H G$ state diagram for closed operators [G3; 66], $J^{\prime} T^{\prime} Q^{\prime}=(Q T J)^{\prime} \in I I_{2} \cup I I I_{2}$. But $R\left(J^{\prime} T^{\prime} Q^{\prime}\right)=R\left(J^{\prime} T^{\prime}\right)$ by Lemma 1.13 and therefore $R\left(J^{\prime} T^{\prime}\right)$ is not closed; in particular, $J^{\prime} T^{\prime} \notin 1 e$ which implies by Theorem 2.2 that $T^{\prime} \notin 1 e$ (since $J^{\prime} \in 1 e$ and $\left.J^{\prime} T^{\prime}=(T J)^{\prime} \neq 0\right)$.

2.6. Proposition. Let $T$ be closed and let $X$ be reflexive. Then $T \in 2 e$ implies $T^{\prime} \notin I I I e$.

Proof. Let $T \in 2 e$. There exists a closed finite codimensional subspace $E$ such that $E \oplus N(T)=D(T)$. Write $J=J_{E}^{X}$. Then $T J \in 2$ and hence from the $T H G$ state diagram for closed operators and Lemma 1.7, $J^{\prime} T^{\prime} \in I \cup I I$. It follows immediately that $\bar{\beta}\left(T^{\prime}\right)<\infty$, i.e. $T^{\prime} \notin$ IIIe. .

The results of this section are summarized in the diagram below. This configuration is identical to that of II.4.11 of [G3]. 
2.7. Essential state diagram for closed operators.

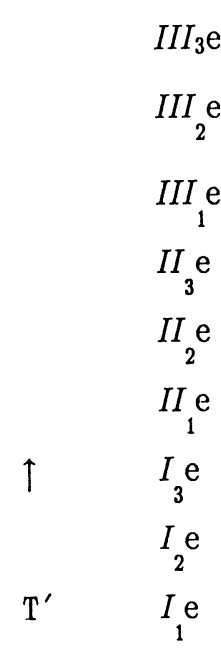

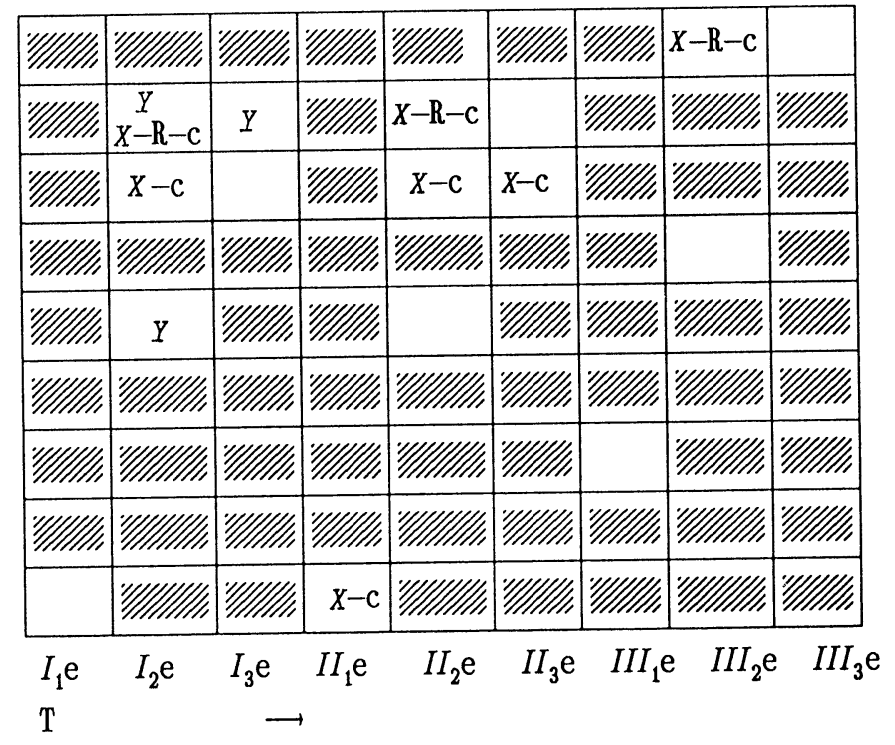

$Y$ : cannot occur if $Y$ is complete,

$X-c$ : cannot occur if $X$ is complete and $T$ is closed, $X-R-c$ : cannot occur if $X$ is reflexive and $T$ is closed.

\section{Completeness of the essential state diagram}

In this section we give examples of essential states which can occur and thus show that the blank squares appearing in the diagram 2.7 all eventuate. We do this by suitably modifying the examples in Section II.5 of [G3]. To simplify the notation we shall for example write $\left(T, T^{\prime}\right) \in\left(I I_{3}, I I I_{1}\right) e$ if $T \in I I_{3} e$ and $T^{\prime} \in I I I_{1} e$. Note that in all the examples of this section $T$ is a bounded operator.

3.1. $T$ bounded, with $X=Y=l_{2}$. $\left(I_{1}, I_{1}\right) e$ : Let $T$ be the identity operator on $X$. $\left(I_{3}, I I I_{1}\right) e$ : Let $T$ be defined by $T\left(\left(x_{k}\right)\right)=\left(x_{2 k}\right)$. Then $T \in I_{3} e$ and $T^{\prime} \in I I I_{1} e$ by 1.17 . $\left(I I I_{1}, I_{3}\right) e:$ Define $T$ by $T\left(\left(x_{k}\right)\right)=\left(0, x_{1}, 0, x_{2}, \ldots\right)$. Then $T \in I I I_{1} e$ and $T^{\prime} \in I_{3} e$ by 1.17. $\left(I I_{2}, I I_{2}\right)$ : The same example as for $\left(I I_{2}, I I_{2}\right)$ in [G3], i.e., $T\left(\left(x_{k}\right)\right)=\left(x_{k} / k\right)$, using 2.7. $\left(I I_{3}, I I I_{2}\right) e$ : The operator $T:\left(x_{k}\right) \rightarrow$ $\left(x_{2 k} / k\right)$ is compact and has dense range. Therefore $T \in I I_{3}$, and then $T^{\prime} \in I I I_{2}$ by 2.7. $\left(I I I_{2}, I I_{3}\right) e$ : Let $T$ be the adjoint of the operator in the previous example. Then $T^{\prime} \in I I_{3}$ e by 2.7. $\left(I I I_{3}, I I I_{3}\right) e$ : Let $T$ be the zero operator. A nontrivial example similar to the corresponding one in [G3] is also easily constructed.

3.2. $T$ compact, with $X=l_{2}$ and $Y$ not complete. $\left(I_{2}, I I_{2}\right) e$ : The same examples as that given for $\left(I_{2}, I I_{2}\right)$ in [G3] serves. $\left(I_{3}, I I I_{2}\right) e$ : Let $T$ be defined by $T\left(\left(x_{k}\right)\right)=\left(x_{2 k} / k\right)$ and let $Y=R(T) \subset l_{2}$. Then $T \in I_{3}$, and since $T^{\prime}$ is compact it is clear that $T^{\prime} \notin 1 e$. Hence $T^{\prime} \in I I I_{2} e$ by 1.17 . 
3.3. $T$ bounded, with $X$ not complete and $Y=l_{2}$. $\left(I_{2}, I I I_{1}\right) e$ : Let $\left\{x_{\alpha}\right\}$ be a Hamel basis for $l_{2}$ and let $X$ be the linear space $l_{2}$ renormed by $\left\|\sum \lambda_{\alpha} x_{\alpha}\right\|=$ $\sum\left|\lambda_{\alpha}\right|$. Let $T$ be the identity map from $X$ onto $l_{2}$. Then $T \in I e$ and is bounded. Suppose $T \in F_{+}$. Let $M$ be a principal subspace of $X$ for which $T / M \in 1$. Then \|\| and the Hilbert norm \|\|$_{2}$ are equivalent on $M$ and hence also on $l_{2}=M \oplus M^{\perp}$ by the fact that any two norms are equivalent on the finite dimensional space $M^{\perp}$, which is clearly impossible. Therefore $T \notin F_{+}$. Consequently $T \in I_{2} e$ and the essential state diagram for closed operators 2.7 now gives $T^{\prime} \in I I I_{1} e$.

$\left(I I_{1}, I_{1}\right) e:$ see $[\mathrm{G} 3,68] .\left(I I_{2}, I I I_{1}\right) e:$ Similar to the example for $\left(I I_{2}, I I I_{1}\right)$ in [G3; 68]. $\left(I I_{3}, I I I_{1}\right) e$ : Similar to the example for $\left(I I_{3}, I I I_{1}\right)$ in [G3] using the operator $\left(x_{k}\right) \rightarrow\left(x_{k}\right) \rightarrow\left(x_{2 k}\right)$ in place of the left shift operator.

3.4. $T$ compact, $X$ complete but not reflexive, $Y=l_{2}$. $\left(I I_{2}, I I I_{2}\right) e$ : The same example in [G3] for $\left(I I_{2}, I I I_{2}\right)$ serves. $\left(I I I_{2}, I I I_{3}\right) e$ : Similar to the example for $\left(I I I_{2}, I I I_{3}\right)$ in $[\mathrm{G} 3]$ but using the operator $\left(x_{n}\right) \rightarrow\left(0, x_{1}, 0, x_{2}, \ldots\right)$ (on $\left.l_{1}\right)$ in place of the right shift operator.

3.5. $T$ compact, $X$ complete but not reflexive, $Y$ not complete. $\left(I_{2}, I I I_{2}\right) e$ : The same example as for $\left(I_{2}, I I I_{2}\right)$ of [G3].

A construction of S. Goldberg [G2] reducees the $T H G$ diagram for closed operators to the bounded case. A similar reduction using [G2] carries through for the "essential" classification. Goldberg's construction has recently been extended to arbitrary operators by L.E. Labuschagne [L].

\section{Uniqueness character of the essential state diagram}

The essential state diagram was achieved by partitioning the class $\{T: \alpha(T)$ $<\infty\}$ into two subclasses, namely the $F_{+}$and non $F_{+}$-operators. We do not claim that our classification is the only one resulting in the same configuration as the $T H G$ model. However, the following alternative classification seems worthy of comment.

1a: The class of operators $T$ for which $(T / M)^{-1}$ exists and is continuous on some principle subspace $M$ complementary to $N(T)$.

2a: The complement of $1 \mathrm{a}$ in the class of $\{T: \alpha(T)<\infty\}$.

The states we now consider are $I e, I I e, I I I e, 1 a, 2 a, 3 a$. The example below shows that this classification results in a different configuration to that of the THG model.

4.1. Example. There exists $T \in I e \cap 2 a$ such that $T^{\prime} \in I e \cap 1 a$.

Let $Y$ be an infinite dimensional Banach space, let $f$ be a discontinuous linear functional on $Y$ and let $T: Y_{f} \rightarrow Y$ be the graph operator associated with $f$. Set $X=Y_{f}$. Then $T^{-1}$ is discontinuous. However $T^{-1} / N(f)$ is an isometry and hence $T \in F_{+}$. Thus $T$ is an injective and surjective $F_{+}$-operator, and $T \in I \cap 2 a$. The essential state diagram 2.7 now gives $T^{\prime} \in I e$ while $T H G$ gives $T^{\prime} \in 1$. Therefore $T^{\prime} \in I e \cap 1 a$. 


\section{References}

[C1] Cross, R.W.: On the continuous linear image of a Banach space. - J. Austral. Math. Soc. Ser. A 29, 1980, 219-234.

[C2] Cross, R.W.: Properties of some norm related functions of unbounded linear operators. - Math. Z. 199, 1988, 285-302.

[C3] Cross, R.W.: Unbounded linear operators of semi-Fredholm type in normed spaces. Portugal. Math. 47, 1990, 61-79.

[C4] Cross, R.W.: On the perturbation of unbounded linear operators with topologically complemented ranges. - J. Funct. Anal. (to appear).

[C5] Cross, R.W.: Linear transformations of Tauberian type in normed spaces. - Note Mat. (to appear).

[GohK] Gohberg, I.C., and M.G. Krein: Fundamental theorems on deficiency numbers, root numbers, and indices of linear operators. - Uspekhi Mat. Nauk 12, 1957, 43-118 (Russsian; translated in Amer. Math. Soc. Transl. 13).

[G1] Goldberg, S.: Linear operators and their conjugates. - Pacific J. Math. 9, 1959, 69-79.

[G2] GoldBERG, S.: Closed linear operators and associated continuous linear operators. - Pacific J. Math. 12, 1962, 183-186.

[G3] Goldberg, S.: Unbounded linear operators. - McGraw-Hill, New York, 1966.

[Ka] Kato, T.: Perturbation theory for nullity, deficiency and other quantities of linear operators. - J. Analyse Math. 6, 1958, 273-322.

[L] LABUSChaGNe, L.E.: Characterisations of partially continuous strictly cosingular and $\varphi_{-}$-type operators. - Glasgow Math. J. (to appear).

[O] OnIEva, V.M.: Adjoint operators and state diagrams in functional analysis. - University of Santander, 1980 (Spanish).

[P] Pietsch, A.: Operator ideals. - North-Holland, Berlin, 1978.

[TH] TAYlor, A.E., and C.J.A. Halberg: General theorems about a linear operator and its conjugate. - J. Reine Angew. Math. 198, 1957, 93-111.

University of Cape Town

Department of Mathematics

Private Bag

Rondebosch 7700

South Africa

Received 20 January 1989 